\title{
Optimum Number of Transistors in Stacked CMOS Millimeter-Wave Power Amplifiers
}

\author{
Mohammad Hassan Montaseri, Janne Aikio, Timo Rahkonen, Aarno Pärssinen \\ Centre for Wireless Communications and Circuits and Systems Research Group \\ Faculty of Information Technology and Electrical Engineering (ITEE) \\ University of Oulu \\ Oulu, Finland \\ \{Firstname.Lastname@oulu.fi\}
}

\begin{abstract}
This paper proposes how to define the optimum number of stacked transistors in a multi-stacked CMOS power amplifier (PA) topology, based on several physical as well as circuit design aspects. Starting with a systematic concept, the analysis then goes through the relevance of transistor transconductance, aspect ratio, parasitics, operating frequency, and number of transistor stages in a pentagonal trade-off concept. While this is done based on theoretical circuit analysis, the results, then, were evaluated using simulations based on 45nm CMOS technology.
\end{abstract}

Keywords-CMOS; millimeter-wave integrated circuits; millimeter-wave power amplifiers; multi-stacked topology;

\section{INTRODUCTION}

With the growth of millimeter-wave spectrum applications the need for fully integrated efficient millimeter-wave power amplifiers (PA) is inevitable. Higher integration capability and lower fabrication cost offered by CMOS technology, has made PA integration a highly desirable feature and challenging target for research. The main drawback of scaled CMOS transistors, however, is their low junction breakdown levels, making them inappropriate for power amplification applications if used in a single transistor topology. Stacking CMOS transistors in series connection is an approach to prevail the foregone problem [1]-[4] in a way that with $N$ transistors, the overall structure can tolerate $N$ times the drainsource breakdown voltage of a single device without violating any transistor junction. This is possible in IC processes, like SOI, where bulk can be isolated properly from the substrate and connected directly to source. Nevertheless, the presence of transistor parasitics dictate phase rotation along each and every stage in stacked transistor topologies; if not tolerable, additional matching networks are necessary so as to both match each stage to its successor and to phase align each single amplifier stage in order for the signals to be efficiently summed up to the output node [5]-[7]. Accordingly, the number of stacked transistors remains limited regardless of either approach, the definition of which is the aim of this paper.

\section{STACKED CMOS POWER AMPLIFIERS}

As shown in Fig. 1, a stacked-transistor PA is composed of series connection of several transistors on top of each other,

This research has been sponsored by InfoTech Oulu Doctoral Program, Finnish Funding Agency for Technology and Innovation (Tekes), Nokia, Esju and CoreHW. where $V_{m}$ is the drain-source amplitude of each transistor. As mentioned earlier, if superimposed efficiently, this results in output voltage swing increase across the overall device to an order of $N \times V_{m}$ whilst none of the junctions experiences any stress. On the other hand, the design requirements mandate each stacked transistor to provide a resistive load of $R_{\text {opt }}$, in a way that the series connection of all stacked transistors is equal to the load to be driven, i.e. $R_{L}$. This is done through dimensioning capacitances $C_{2}$ to $C_{N}$ using either approaches proposed in [4], [5], [7], or [8].

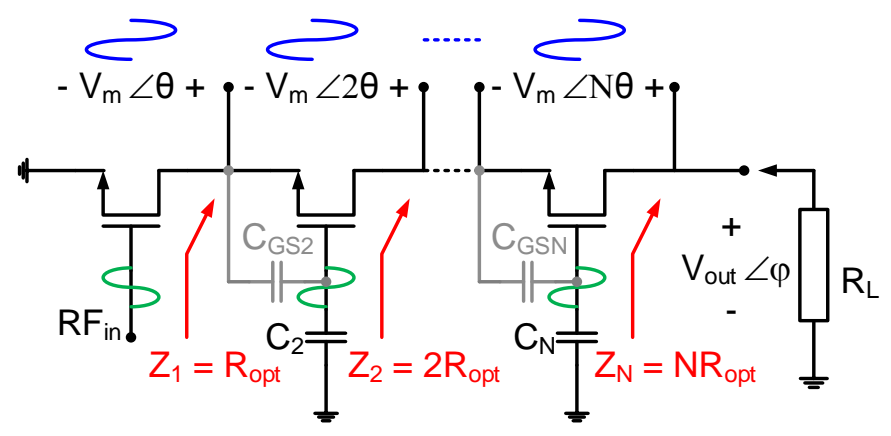

Fig. 1. Multi-input multi-output modeling of multi-stacked transistors PAs.

Due to the presence of parasitics, the angles of drainsource voltage vectors tend to gradually rotate per stacked transistor along the device. For example, in case of uniform phase rotation, summing all drain-source voltages yields the amplitude of the output of interest as

$$
\left|V_{\text {out }}\right|=\left|\sum_{n=1}^{N} V_{m} e^{j n \theta}\right|=V_{m} \times \frac{\sin (N \theta / 2)}{\sin (\theta / 2)}
$$

From (1) the dependence of maximum output amplitude on the phase rotation posed by each stage can be demonstrated. Indeed, inter-stage phase misalignment results in radio frequency (RF) signal amplitude to destructively depart from its ideal magnitude (see Fig. 2) and hence output power and efficiency reduction.

It can be seen from Fig. 2 that the maximum swing degrades from ideal case with phase rotation per stage. In addition, due to excess phase rotation, after a certain number of transistor stages, the maximum amplitude starts to saturate reaching an absolute maximum and decreases afterwards. It 
should be noted that this saturation is due to the fact that excess phase rotation gradually reduces amplitude of the previous stages.

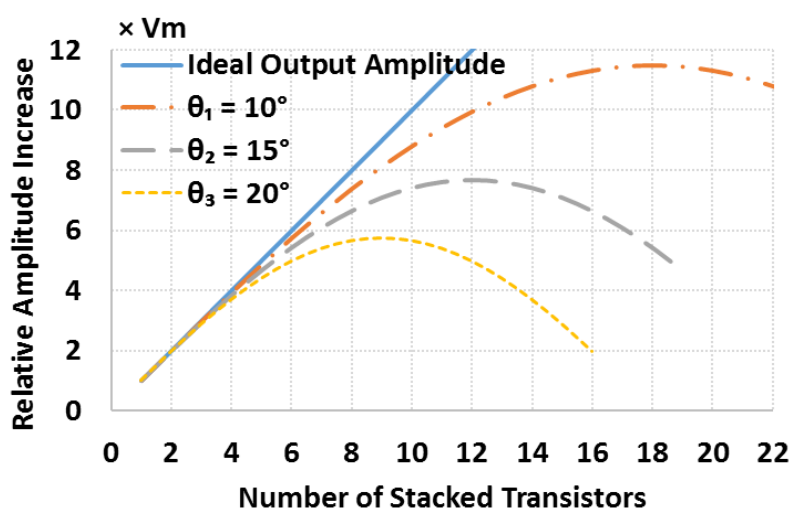

Fig. 2. The impact of phase rotation on the output maximum amplitude of stacked-transistor PAs.

A closer look at (1) in polar plot reveals that the total phase rotation should be bound in practice to about $120^{\circ}$ instead of $180^{\circ}$. This is because after this amount of phase shift the increase in signal amplitude is rather very low. For example, consider a phase rotation of $10^{\circ}$ per stacked transistor; the first 12 stages reach a level of $10 \times V_{m}$ while the next 6 stages will only add approximately $2 \times V_{m}$ more which leads to an approximately $64 \%$ energy loss compared to optimum case. By the same token, for $\theta=15^{\circ}$ and $\theta=20^{\circ}$ the optimum numbers of stages are 8 and 6 , respectively; while the remaining stages, i.e. from $120^{\circ}$ up to $180^{\circ}$, will only add approximately $1-1.3$ times $V_{m}$, which yields drastic efficiency degradation and must be avoided. Thus, $120^{\circ}$ can be regarded as a sweet spot of the stacked transistor PAs design.

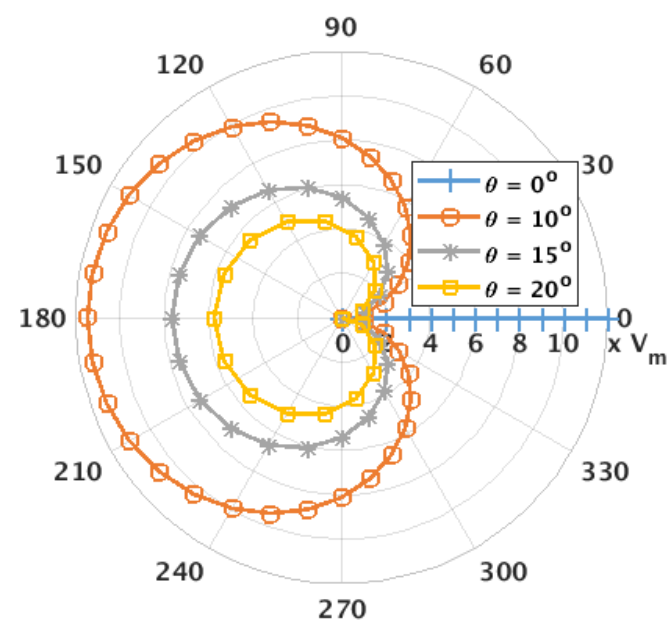

Fig. 3. Gradual growth of the amplitude of $n$ stacked transistors with respect to different superposition angle.

Since phase rotation stems from the presence of parasitics, it can be defined from the input impedance of each stage using the MOS transistor model at high frequencies as shown in Fig. 4.

Analytical model gives the input impedance of the $\mathrm{n}^{\text {th }}$ stage to be

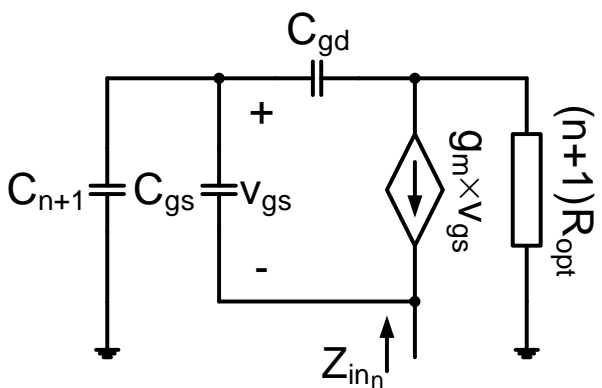

Fig. 4. MOS transistors at high frequencies using Mayer parasitic capacitances model.

$$
Z_{i n_{n}} \approx \frac{C_{g s}+C_{g d}\left(1+g_{m}(n+1) R_{o p t}\right)+C_{n+1}}{\left(g_{m}+s C_{g s}\right)\left(C_{g d}+C_{n+1}+s(n+1) R_{o p t} C_{n+1} C_{g d}\right)},
$$

where $R_{\text {opt }}$ is the optimum load of each single transistor and $C_{n}$ is the designed gate capacitance of common gate stages.

Apparently (2) reveals phase variation as a direct consequence of the presence of frequency dependent terms in the input impedance. Thus the amount of phase variation of each stage can be calculated as

$$
\theta_{n}=\tan ^{-1}\left((n+1) \omega R_{o p t}\left(C_{g d} \| C_{n+1}\right)\right)+\tan ^{-1}\left(\frac{\omega C_{g s}}{g_{m}}\right)
$$

and the total phase variation can be defined to be

$$
\theta_{t o t}=\sum_{n=1}^{N}\left(\tan ^{-1}\left((n+1) \omega R_{o p t}\left(C_{g d} \| C_{n+1}\right)\right)+\tan ^{-1}\left(\frac{\omega C_{g s}}{g_{m}}\right)\right)
$$

Substituting the method explained in [5] for dimensioning $C_{n}$ and using "Euler-Maclaurin" summation method, on the condition that $\omega R_{o p t}\left(C_{g d} \| C_{n}\right)$ and $\omega C_{g s} / g_{m}$ are less than 1 , (4) can be approximated as

$$
\theta_{t o t} \approx \frac{(N+1) \omega R_{L} C_{g d}\left(C_{g s}+C_{g d}\left(1+g_{m} R_{L}\right)\right)}{2\left(C_{g s}+g_{m} R_{L} C_{g d}\right)}+\frac{N \omega C_{g s}}{g_{m}} .
$$

Regarding $120^{\circ}$ as the total phase rotation threshold for the design, (4) can be solved for the optimum number of stacked transistors as follows

$$
\frac{(N+1) \omega R_{L} C_{g d}\left(C_{g s}+C_{g d}\left(1+g_{m} R_{L}\right)\right)}{2\left(C_{g s}+g_{m} R_{L} C_{g d}\right)}+\frac{N \omega C_{g s}}{g_{m}}=\frac{2 \pi}{3} .
$$

Equation (6) predicts the optimum number of stacked transistors for which the input impedance of $n+1^{\text {st }}$ stage, i.e. the load impedance of $n^{\text {th }}$ stage, starts to destruct the drain node voltage hence overall device output power and efficiency. It should be noted that (5) reveals several 
interesting design trade-offs as well. Accordingly, it can be deduced that any increase in phase yields reduction in the number of transistor stages. More importantly, phase varies in direct proportion to the operating frequency, transistor parasitics, and the load line, while indirectly to transconductance of the transistors. The transconductance, however, is defined both by transistor biasing and aspect ratio. This gives a trade-off between the mentioned design parameters shown in Fig. 5.

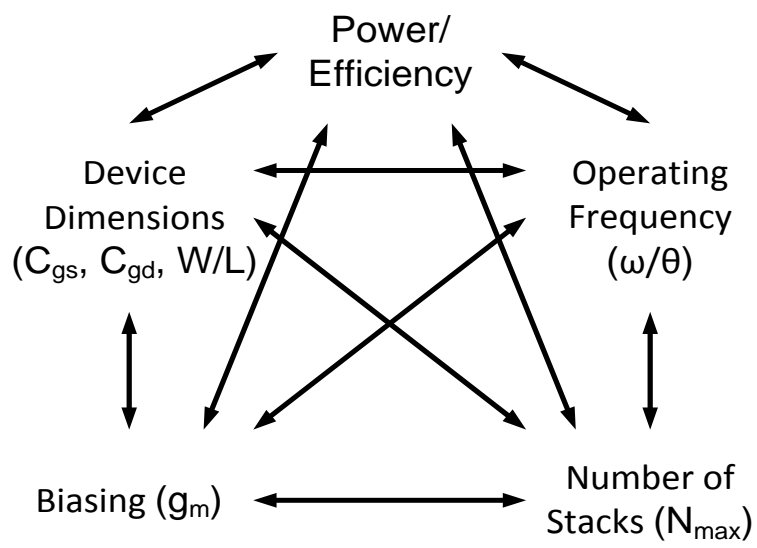

Fig. 5. Multi-stacked transistors PAs design trade-offs.

\section{Simulation Results}

In order to evaluate the theoretical analysis presented in previous section, a multi-stacked-transistor PA (Fig. 6) was designed to supply $100 \mathrm{~mW}$ into a load of $R_{L}=50 \Omega$ with a $25 \mathrm{~dB}$ voltage gain at $28 \mathrm{GHz}$ operating frequency, based on 45nm CMOS technology. $R_{1}-R_{N+1}$ are designed to be much higher compared to the $C_{n}$ reactances at the desired frequencies.

Based on the requirements, transistor biasing and dimensions were designed such that their transconductance $g_{m}$, gate-source capacitance $C_{g s}$, and gate-drain capacitance $C_{g d}$ were $350 \mathrm{mS}, 200 \mathrm{fF}$, and $45 \mathrm{fF}$, respectively. Substituting the given parameters in (6) and solving for the number of stacked transistors $N$ gives 10.5 . Since this is the number of transistors for which a $120^{\circ}$ phase rotation takes place, it is recommended to round 10.5 down to 10 transistors for the structure, i.e. an approximately average of $12^{\circ}$ of phase rotation per stage. Fig. 7 depicts the simulated phase rotation of each single stage as well as cumulative phase rotation of each added stage compared to the proposed theoretical formulae (3) and (5), respectively.

From (6) it can be seen that while the analysis predicts the optimal number of stacked transistors to be ten, simulation results reveal that it is roughly eight. This indicates that the average phase rotation per stage is somewhat more than what was calculated, i.e. almost $16^{\circ}$ per stage. Thus, the proposed analytical calculations comply fairly well with the simulation results. However, their difference stems firstly from the fact that some approximation has been made in calculating (5), and secondly the parasitics (capacitances, resistances, etc.) are nonlinear in their nature; since they tend to vary with signal level, AM - PM conversion distortion is inevitable.
Fig. 8 shows a plot of maximum efficiency, $\eta_{\max }$, as a function of the number of the stacked transistors. As mentioned earlier, due to phase misalignment of the stages, the output voltage amplitude deviates destructively from its ideal value. Accordingly, the drain efficiency (DE) starts to degrade.

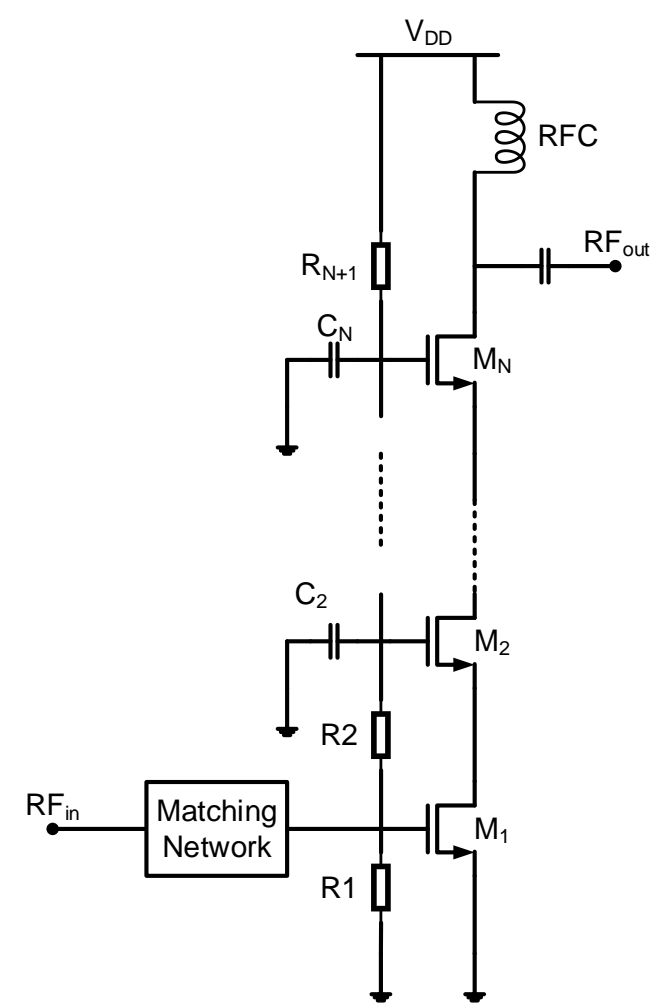

Fig. 6. The designed multi-stacked CMOS PA schematic circuit.

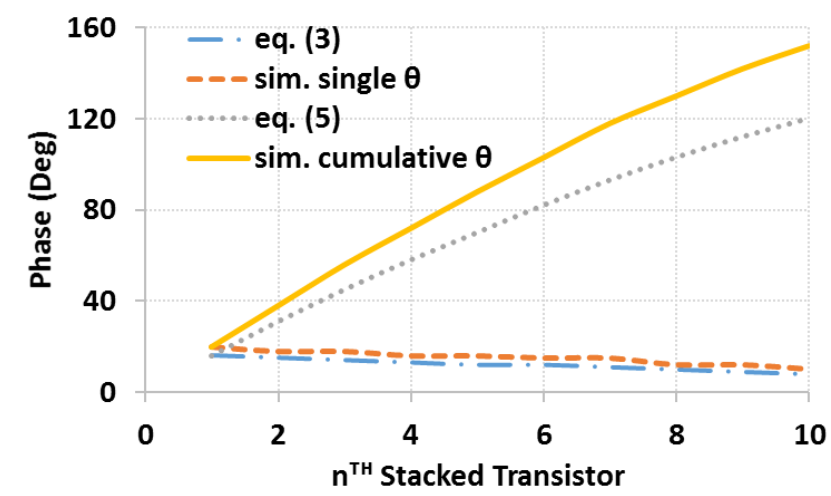

Fig. 7. Single-stage/cumulative phase rotation posed by each stacked transistor.

The rate at which the designed multi-stacked transistor PA loses efficiency per added stage is also plotted in Fig. 8. Firstly, it can be seen that the loss curvature is an exponent of the phase rotation per stacked transistor. Secondly, the steepness of the curvature increases with phase misalignment. In case of excess phase rotation, due to additional stages, the structure may produce RF signals which cancel the preceding stages' resulting in $100 \%$ efficiency loss of that stage; e.g. theoretically the $11^{\text {th }}$ and by simulation the $9^{\text {th }}$ stacked 
transistor will be totally useless if used. This, in addition to previous explanations, supports the analytical calculations, which were proposed in previous section. In this example, eight transistor stacks can be considered if maximum power delivering capability is sought. However, that already reduces drain efficiency more than $10 \%$ compared to optimum class-A performance. In case of maximum efficiency, only stack of 3...4 transistors should be used.

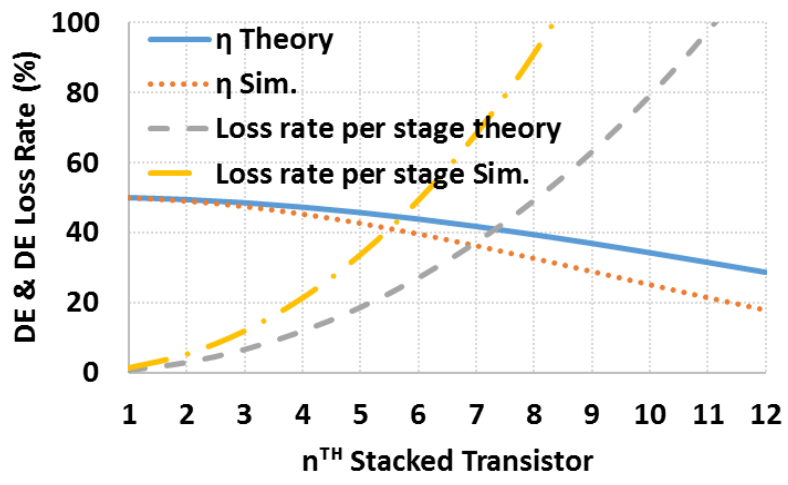

Fig. 8. Drain efficiency (DE) of the overall PA device per number of stacked transistors and efficiency degradation rate of each stacked transistor.

Fig. 9 shows simulated small signal s-parameters of the 8stack transistor PA. It can be seen that the desired voltage gain is achieved. Also shown in Fig. 10 are output power, power gain, and drain efficiency (DE) of the PA. The designed PA provides a $20 \mathrm{dBm}$ of saturated power along with a maximum drain efficiency of $32 \%$.

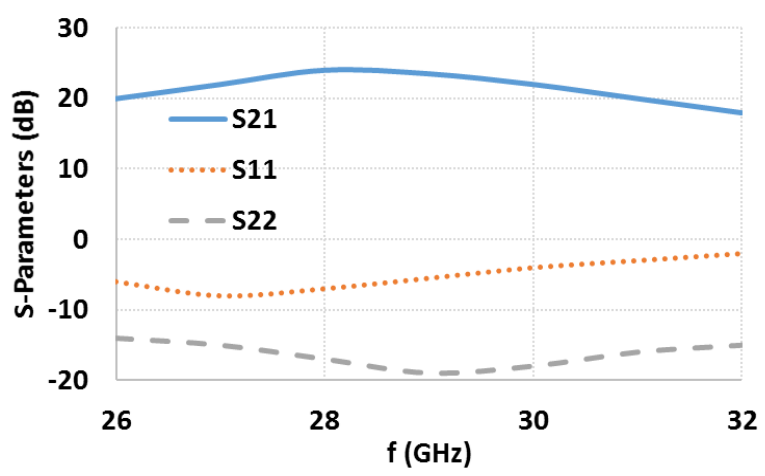

Fig. 9. Simulated s-parameters of the designed stacked CMOS PA.

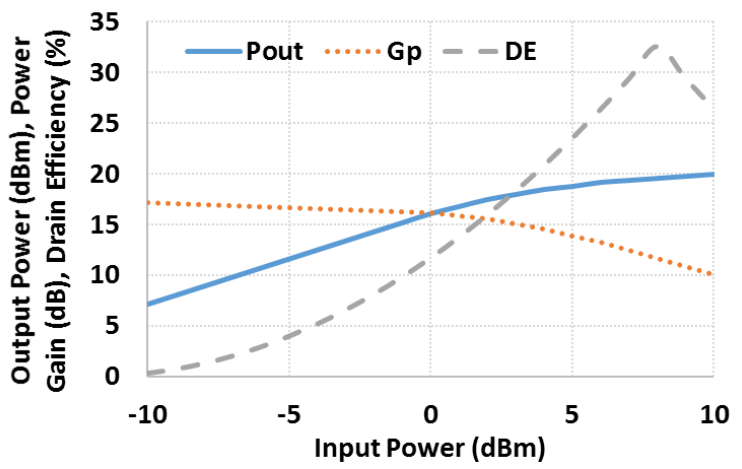

Fig. 10. Simulated output power, power gain, and drain efficiency (DE) of the designed stacked CMOS PA.

\section{CONCLUSION}

In this paper, a theoretical method was developed to define the maximum/optimum number of stacked transistors in multi-stacked transistor PAs using several theoretical formulae. The study started from a systematic viewpoint up to transistor level physical parameters. It was shown how the operationg frequency, and transistor aspect ration, through gate-source and gate-drain capacitances, and transconductance through biasing, define the maximum as well as the optimum number of stacked transistors. It was justified that any increase in either or both operating frequency and parasitic capacitances will translate into phase rotation, which in turn reduces the maximum allowed number of transistor stages. Conversly, increasing transconductance of transistors results in phase reduction while allowing some room for either more device stacking or higher operating frequency. However, it should be noted that this is in a compromise with the transistor dimensioning.

Based on the presented analysis, a CMOS PA was designed using 45nm CMOS technology. An optimum number of 10 stacked transistors was calculated analytically at $28 \mathrm{GHz}$ operating frequency. According to simulations $20 \mathrm{dBm}$ saturated output power with drain efficiency of $32 \%$ could be reached. This acounted for $10 \%$ discrepancy between simulation and analysis. However, the simulation results were in good agreement with the analytical calculations which proves the validity of the proposed method.

\section{ACKNOWLEDGMENT}

The authors would like to express their gratitude towards InfoTech Oulu Doctoral Program.

\section{REFERENCES}

[1] J. Cui, S. Helmi, Y. Tang, and S. Mohammadi, "Stacking the Deck for Efficiency: RF- to Millimeter-Wave Stacked CMOS SOI Power Amplifiers," IEEE Microwave magazine, vol. 17, issue 12, pp. 55 - 69, December 2016.

[2] J.A. Jayamon, J.F. Buckwalter, and P.M. Asbeck, "Multigate-Cell Stacked FET Design for Millimeter-Wave CMOS Power Amplifiers," IEEE Solid-State Circuits J., vol. 51, no. 9, pp. 2027-2039, September 2016.

[3] S.R. Helmi, J. Chen, and S. Mohammadi, "High-Efficiency Microwave and mm-Wave Stacked Cell CMOS SOI Power Amplifiers," IEEE Trans. Microwave Theory and Techniques, vol. 64, no. 7, pp. 2025 2038, July 2016.

[4] Y. Kim, and Y. Kwon, "Analysis and Design of Millimeter-Wave Power Amplifier Using Stacked-FET Structure," IEEE Trans. Microwave Theory and Techniques, vol. 63, no. 2, pp. 691-702, February 2015

[5] H. Dabag, B. Hanafi, F. Golcuk, A. Agah, J.F. Buckwalter, and P.M Asbeck, "Analysis and Design of Stacked-FET Millimeter-Wave Power Amplifiers," IEEE Trans. Microwave Theory and Techniques, vol. 61, no. 4, pp. 1543-1556, April 2013.

[6] A.K. Ezzeddine, H.C. Huang, and J.L. Singer, "UHiFET-A new highfrequency high-voltage device," in IEEE MTT-S Int. Microw. Symp. Dig., 2011, pp. 1-4.

[7] S. Pornpromlikit, "CMOS RF power amplifier design approaches for wireless communications," Ph.D. dissertation, Dept. Elect. Comput. Eng., Univ. California at San Diego, San Diego, CA, USA, 2010.

[8] M. Lei, Z. Tsai, K. Lin, and H. Wang, "Design and analysis of stacked power amplifier in series-input and series-output configuration," IEEE Trans. Microwave Theory and Techniques, vol. 55, no. 12, pp. 2802 2812, December 2007. 